\title{
securePrune:Secure block pruning in UTXO based blockchains using Accumulators
}

\author{
Swaroopa Reddy B \\ Department of Electrical Engineering \\ Indian Institute of Technology Hyderabad \\ Hyderabad, India \\ ee17resch11004@iith.ac.in
}

\begin{abstract}
In this paper, we propose a scheme called securePrune for reducing the storage space of a full node and synchronization time of bootstrapping nodes joining the Peer-toPeer (P2P) network in an Unspent Transaction Outputs (UTXO) based blockchain like bitcoin using RSA accumulators. The size of the bitcoin blockchain is growing linearly with transactions. We propose a new block structure to represent the state of a blockchain also called $U T X O$ set by including an accumulator of a state in the block header and proofs of knowledge for inclusion and deletion of the transactions of the current block in the block. In our scheme, the miners periodically release a snapshot of the blockchain state. The other full nodes in the network, securely prune the historical blocks after attaining the required number of confirmations for the snapshot block, which in turn confirms the snapshot of the state through an accumulator specified in the block header and proofs inside the block. The secure and periodic pruning of the old blocks, reduce the synchronization time for a new node joining into the network. The simulation results demonstrate a significant reduction in the storage space of a full node and bootstrapping cost of the new nodes.

Index Terms-Blockchain, UTXO, RSA Accumulator, Pruning, Inclusive Proofs, Bootstrapping.
\end{abstract}

\section{INTRODUCTION}

The Blockchain is a revolutionary technology behind the Peer-to-Peer (P2P) cryptocurrency networks like bitcoin [1] and smart contract enabled P2P networks like Ethereum [2] and Hyperledger [3]. The decentralized and trust less nature of the blockchain created a space for applications in healthcare [4] , Internet of Things (IoT) [5].

The transactions are the fundamental entities in the blockchain which represents the transfer of coins from one party to another. The miner collects the multiple transactions from other miners/fullnodes and ceates a block with computationally hard problem called Proof-of-Work (PoW) [1]. The immutability of the blockchain ledger lies in PoW, as any modification to the ledger by a miner needs to recompute all the blocks till the current position of the chain. To achieve the consensus among all the nodes, while creating a new node the miner selects a tip of the longest chain in the network.

The bitcoin blockchain is a P2P network of miners [1], fullnodes [6] and simplified payment verifiation nodes (SPV) [1]. The miners play a key role in generating the blocks

This research was funded by Indigenous 5G Test Bed (Building an end to end 5G Test Bed) in India, Department of Telecommunication Network \& Technologies (NT) Cell. through PoW puzzle. The full node stores all the blocks since the genesis block along with the blockchain state (UTXO set) [7]. The UTXO set keep track of the all unspent output transactions of the historical blocks and used as sources for new input transactions. A full node contributes to the security of the network through block validation. However, running a full node incurs storage costs as the blockchain data grows exponetially [9] with time. The main advantage of storing the all blocks by the full node is to make the bootstrapping nodes synchronize with the existing network nodes.

Erasure-code based low storage blockchain node is proposed in [12]. These nodes split every block into fixed size data fragments and generates the coded fragments from the linear combination of the random coefficients generated from the pseudo-random generator. The node can reconstruct the block by the inverse linear combinations. The main limitation of this work is that they only consider the case when nodes can leave the network or can be unreachable, they do not consider adversarial nodes that can provide maliciously formed coded fragments.

A Secure Fountain architecture founded on coding theory is proposed in [13] for storage efficiency of the blockchain node by reducing the storage cost and still contribute to bootstrap a new node joining the network. In this scheme, the nodes reduce the storage cost by encoding the validated blocks into a small number of coded blocks using founain codes [14]. The secure Fountain $(\mathrm{SeF})$ archetecture uses header-chain as a side information to check whether the decoded blocks are formed from the mallicious modifications.

In [15], authors proposed a Dynamic distributed storgae for scaling the blockchains by allocating the nodes into dynamic zones. The nodes in each zone will store a share of private key using shamir's secret sharing [16] for encrypting the block data and apply a distributed storage codes such as [17], [18] for reducing the storage cost.

However, In all these works, a bootstrapping node need to download all the blocks in the form of distributed coded fragments and validate all the decoded blocks to synchronize with the existing nodes.

In this paper, we propose a periodic pruning of the historical blocks based on the security confirmations guaranteed by the RSA accumulator [10], [11] of the UTXO set and PoW based longest chain consensus algorithm [1]. 
The main contributions of the paper are as follows.

1) The algorithm for block generation by a miner by adding accumulator for state (UTXO set) in the block header to make the state as a part of the PoW consensus algorithm. The miner also includes Non-Interactive proof of Exponentiation (NI-PoE) proofs for inclusion of the new output transations and deletion of the UTXO sources of the input transactions of the current block.

2) The algorithm for validation of a block by every full node based on the NI-PoE proofs [11] added by the miner inside the block.

3) The periodic pruning of the blocks at regular intervals of the block height based on the security guaranteed by the accumulator state and the NI-PoE proofs.

4) The bootstrapping procedure for synchronizing the new nodes joining the network.

Through the event-driven simulation of blockchain, we have shown the $85 \%$ reduction in the storage space of a securePrune protocol full node compared to the bitcoin full node and also significant reduction in the synchronization time due to the requirement of validation of less number of historical blocks compared to the validation of all the historical blocks in the bitcoin.

The rest of the paper is organized as follows. In Section II, we present the related work in the reduction of the storage space of a bitcoin full node. Section III gives the system model and notations used in the protocol. Section IV describes the preliminaries for generation of accumulator state and NIPoE proofs. In Section V, we decribe the proposed protocol for secure and periodic pruning and synchronization of the bootstrapping nodes. In Section VI, we present and discuss the simulation results. Section VII presents the concluding remarks and future works.

\section{RELATED WORK}

The SPV node or light weight node [1] keep the block headers of a longest PoW chain, instead of the entire blockchain data. The lightweight node depends on the full node for the verification of a transaction by querying the merkle branch linking the transaction to block where it is time stamped. The block pruning [6] is allowed in bitcoin to store most recent blocks of the chain. However, due to lack of old blocks like in full node, pruned node cannot serve the new full nodes joining the network. The concept of assumed-valid blocks [19] have been introduced in bitcoin, where a bootstrapping node skips the script validation of the transactions for ancestors of knowngood blocks, without changing the security model. However, the new nodes still need to download entire historical data to create the current state of the blockchain.

In coinPrune [20] protocol, the authors proposed the pruning older blocks by creating a snapshot of the state at regular intervals, provided the collective reaffirmations to snapshot by the miners. In this protocol, the fullnode prunes the historical blocks provided that the snapshot receives the required number of reaffirmations from all the miners. However, there is a possibilty of the Denial-of-Service (DOS) attack by the
TABLE I:

Parameters used in the securePrune protocol

\begin{tabular}{|c|c|}
\hline Symbols & Description \\
\hline hash $()$. & Cryptographic hash function \\
\hline $\operatorname{root}()$. & Merkle root of set of transactions \\
\hline$H_{\text {prime }}()$. & Prime representative function \\
\hline validate(.) & Transaction validation function \\
\hline$R_{i}$ & Merkle root of $t_{i}$ \\
\hline$B_{i}$ & $i^{\text {th }}$ block of the blockchain \\
\hline$M$ & Memory pool/set of unconfirmed transactions \\
\hline$t x$ & Transaction \\
\hline$t_{i}$ & $\left(t x_{1}, t x_{2}, \ldots, t x_{\left|t_{i}\right|}\right):$ Set of transactions in $B_{i}$ \\
\hline$S_{i}$ & State of the block chain (UTXO set) at block $B_{i}$ \\
\hline$S_{d}$ & Set of utxo's spent in the new block \\
\hline$S_{a}$ & Set of output transactions in the new block $B_{i}$ \\
\hline$A_{i}$ & Accumulator state \\
\hline$W$ & List of membership witnesses of $U T X O$ set \\
\hline$\overline{\pi_{d}}$ & NI-PoE proof for deletion of set $S_{d}$ from accumulator \\
\hline$\pi_{a}$ & NI-PoE proof for addition of set $S_{a}$ to accumulator \\
\hline$T_{\text {proofs }}$ & NI-PoE verification time \\
\hline$\Delta_{s}$ & Number of blocks between two snapshots \\
\hline$n$ & Number of nodes in the network \\
\hline$q$ & Fraction of the attacker's hashrate \\
\hline$n_{p}$ & Number of peers connected to each node \\
\hline$T_{p}$ & Propagation delay \\
\hline$b$ & Block size in $\mathbf{M B}$ \\
\hline$R$ & Average download bandwidth \\
\hline$R_{v}$ & Average validation rate of a block \\
\hline$p_{v}$ & Computational power with node $v$ \\
\hline$\lambda$ & Block creation rate \\
\hline$D$ & End-to-end delay in the network \\
\hline$k$ & Number of confirmations \\
\hline$h$ & Height of the blockchain \\
\hline$m$ & Number of mining nodes in the network \\
\hline
\end{tabular}

miners in reaffirming the snapshot. So, theere is no gaurantee that pruning will happen at every reaffirmation window of a snapshot release.

\section{SySTEM MODEL AND PARAMETERS}

The parameters used in securePrune protocol are listed in TABLE 【.

\section{A. Overview of the transactions and UTXO set}

There are two types of the transactions in every transaction of a block - inputs and outputs [1]. The inputs specifies the previous transaction outputs as sources of the bitcoins in a transaction and the outputs are the destination of bitcoin transfer. Each transaction contains multiple inputs and multiple outputs to combine and split the values of the coin transfer. The full node stores the $U T X O$ set in the chainstate database of the Bitcoin core [8]. The database consists of records of key-value pairs [7]. The key of the record is transaction hash and the value stores the transaction information. Every record 
in the UTXO set represent the outputs yet to be spent in future transactions.

Let at a block height $i$, every full node in the blockchain stores a copy of the state (UTXO set) $S_{i}$ repersented as

$$
S_{i}=\left\{u_{j}: j=1,2, \ldots,\left|S_{i}\right|\right\}
$$

Where, $u_{j}$ is a record in the UTXO set

However, for every new block $\left(B_{i}\right)$ addtion to the chain, the state of the full node changes with the transaction set $t_{i}$ as described in state transition Algorithm in Section V-A1

\section{B. The modified block structure in the proposed protocol}

The blockchain at a height $h$ is modeled as a vector of blocks represented as

$$
C_{h}=\left(B_{0}, B_{1}, \ldots, B_{h}\right)
$$

where, each block $B_{i}$ is a tuple consists of block header $\left(H_{i}\right)$, NI-PoE proof $\left(\pi_{d}\right)$ for deletion of set $S_{d}$ from the UTXO set and NI-PoE proof $\left(\pi_{a}\right)$ for addition of set $S_{a}$ to the UTXO set. While generating a new block, the miner includes a list of transactions $t_{i}$ into the block from the transaction memory pool $(M)$ stored with every miner and full nodes.

$$
B_{i}=<H_{i},\left(A_{i-1}^{\prime}, \pi_{d}, \pi_{a}\right), t_{i}>
$$

Where, the tuple $\left(A_{i-1}^{\prime}, \pi_{d}, \pi_{a}\right)$ results from the state transition of the UTXO set.

In addition to the elements of the bitcoin block header, our proposed model includes an extra element called the accumulator state $\left(A_{i}\right)$, which is an RSA accumulator [10] to represent the sate of the blockchain $\left(S_{i}\right)$ in the block header.

$$
H_{i}=\left(h_{i-1}, \text { nonce, } A_{i}, x\right)
$$

where, $h_{i-1}=\operatorname{hash}\left(H_{i-1}\right)$, nonce is a variable to solve the PoW puzzle and $x$ is the other meta data (like version, time, difficulty etc) similar to bitcoin block header [1]. The modified structure of the block is shown in Fig. 1

\section{PRELIMINARIES}

The following definitions of RSA accumulators [10], [11] are used in our work.

Definition 1. (Accumulator of State). Let $\mathbb{G}$ be a group of unknown order and $g \in \mathbb{G}$, the accumulator state of a block $B_{i}$ is an RSA accumulator [10] of the unspent transaction outputs present in $U T X O$ set $S_{i}=\left\{u_{j}: j=1,2, \ldots,\left|S_{i}\right|\right\}$ and is computed as

$$
A_{i}=\prod_{j=1}^{\left|S_{i}\right|} g^{U_{j}}
$$

Where, $U_{j}$ is the prime representative of the element $u_{j}$ [11]

$$
U_{j}=H_{\text {prime }}\left(u_{j}\right)
$$

The dynamic accumulator [21] is an accumulator that allows to add or delete elements to the accumulator.

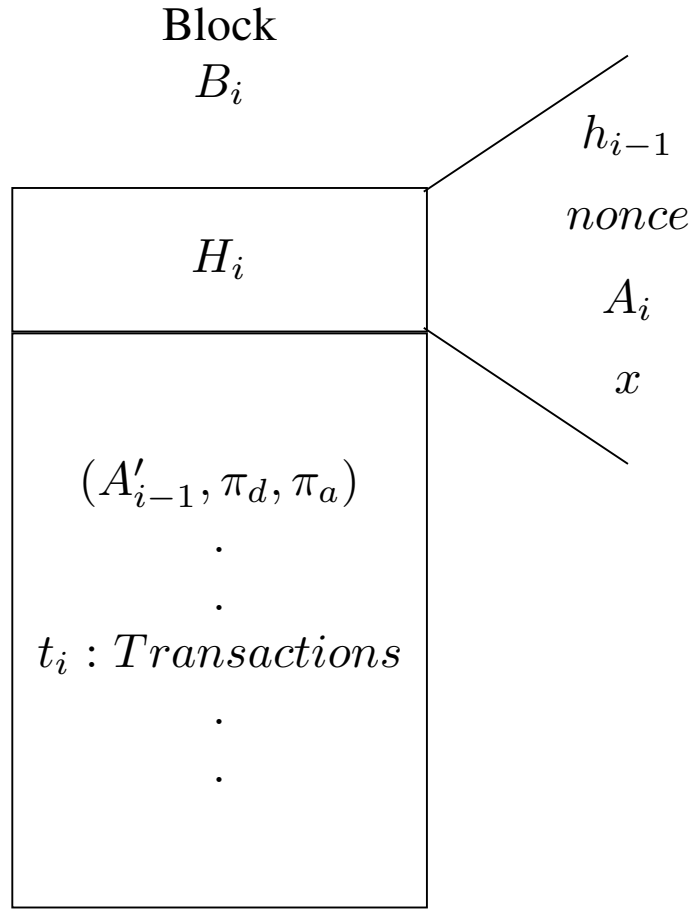

Fig. 1: securePrune Block structure

Definition 2. (Membership Witness). A membership witness is simply an accumulator without an aggregated element. The membership witness for $u_{m}$ is defined as

$$
W_{m}=\prod_{j=1, j \neq m}^{\left|S_{i}\right|} g^{U_{j}}
$$

Definition 3. (Shamir Trick). Let $x, y \in S$ and $g \in \mathbb{G}$, the membership witnesses for $x$ and $y$ are the $x^{\text {th }}$ root of $g$ and $y^{t h}$ root of $g$, then the ShamirTrick [22], [11] is a $(x y)^{t h}$ root of the group element $g$ from the Bezout's coefficients of $x$ and $y$.

While creating a new block, the miner generates new accumulator state $A_{i}$ from $A_{i-1}$ in two stages - The deletion of the set $S_{d}$ from the accumulator $A_{i-1}$ followed by addition of set $S_{a}$ to obtain the new state $A_{i}$.

$$
A_{i}=\operatorname{Batch} A d d\left(\operatorname{BatchDel}\left(A_{i-1}, S_{d}\right), S_{a}\right)
$$

Definition 4. (Batch Deletion (BatchDel)). Let $S_{d}$ represent the set of sources for inputs of the transactions in the new block $B_{i}$, the state $S_{i-1}$ needs to delete the records $S_{d}$ from the database. The deletion of the set $S_{d}$ from accumulator state $A_{i-1}$ can be obtained from BatchDel [11]. The BatchDel uses the membership aggregation function $A g g M e m W i t$ [11] to compute the aggregate membership witness of all elements in $S_{d}$ from the individual membership witnesses of each element. The AggMemWit is simply an accumulator without elements of set $S_{d}$.

$$
A_{i-1}^{\prime}=W_{a g g}=\prod_{u_{j} \in S_{i-1} \backslash S_{d}} g^{U_{j}}
$$


where, $W_{a g g}$ is the aggregated membership witness of all the elements of the set $S_{d}$ generated by Shamir Trick [11]. The BatchDel gives the intermediate state of the accumulator $A_{i-1}^{\prime}$ to process further for obtaining the new accumulator state $\left(A_{i}\right)$ of the new block $B_{i}$ from the set $S_{a}$.

Definition 5. (Batch Addition (BatchAdd). The addition of the elements of set $S_{a}$ to accumulator state requires a batch addition BatchAdd [11] for efficient computation.

$$
A_{i}=\left(A_{i-1}^{\prime}\right)^{U^{*}}
$$

where,

$$
U^{*}=\prod_{s \in S_{a}} H_{\text {prime }}(s)
$$

Definition 6. (Proof of Exponetiation (PoE) [11]). Let $\mathbb{G}$ be a group of unknown order and $u, w \in \mathbb{G}$, the proof of exponetiation in the Group $\mathbb{G}$, when both the prover and verifier are given $(u, w, x \in \mathbb{Z})$ and the prover wants to convince the verifier that $w=u^{x}$.

The Non-interactive PoE (NI-PoE [11]) proofs $\pi_{d}$ and $\pi_{a}$ are generated during the batch updates for the efficient verification without any interaction between prover(miner) and verifier(full node).

$$
\pi_{d}=N I-\operatorname{PoE}(u, x, w)
$$

Definition 7. (Updating membership witnesses). The intermediate accumulator state (9) denotes the membership witness for all the elemets of the set $S_{d}$. Let $s \in S_{i-1} \backslash S_{d}$ and $w_{s}$ is the membership witness of $s$ before deletion of set $S_{d}$ as per (7), then the updated membership witnesses for all $s \in S_{i-1} \backslash S_{d}$ are generated as follows

$$
w_{s}^{\prime}=\operatorname{ShamirTrick}\left(A_{i-1}^{\prime}, w_{s}, \prod_{x \in S_{d}} x, s\right)
$$

The memebrship witness updates for all $s \in S_{i-1} \backslash S_{d}$ after the addition of elements of the set $S_{a}$ are calculated as follows

$$
w_{s}^{\prime \prime}=\left(w_{s}^{\prime}\right) \prod_{x \in S_{a}} x
$$

The membership witnesses for elements $x \in S_{a}$ are calculated as follows

$$
w_{x}=\left(A_{i-1}^{\prime}\right) \prod_{y \in S_{a}, y \neq x} y
$$

\section{SECURE BLOCK PRUNING PROTOCOL}

In this section, we discuss the proposed secure pruning protocol for storage scalability and the synchronization process of the bootstrapping nodes. The protocol requires the modification in the block generation procedure by the miners and the validation procedure of a block by the full nodes in the network based on the accumulators and NI-PoE.

\section{A. Requirements of the securePrune protocol}

1) State transition Algorithm: The UTXO set (state) of the blockchain is dynamic and changed for every new block addition to the blockchain. The following algorithm describes the transition of a miner (or full node) while generating a new block (or after receiving a new block). The new state transition function returns the set of deleted elements $\left(S_{d}\right)$ and added elements $\left(S_{a}\right)$ along with the new UTXO set.

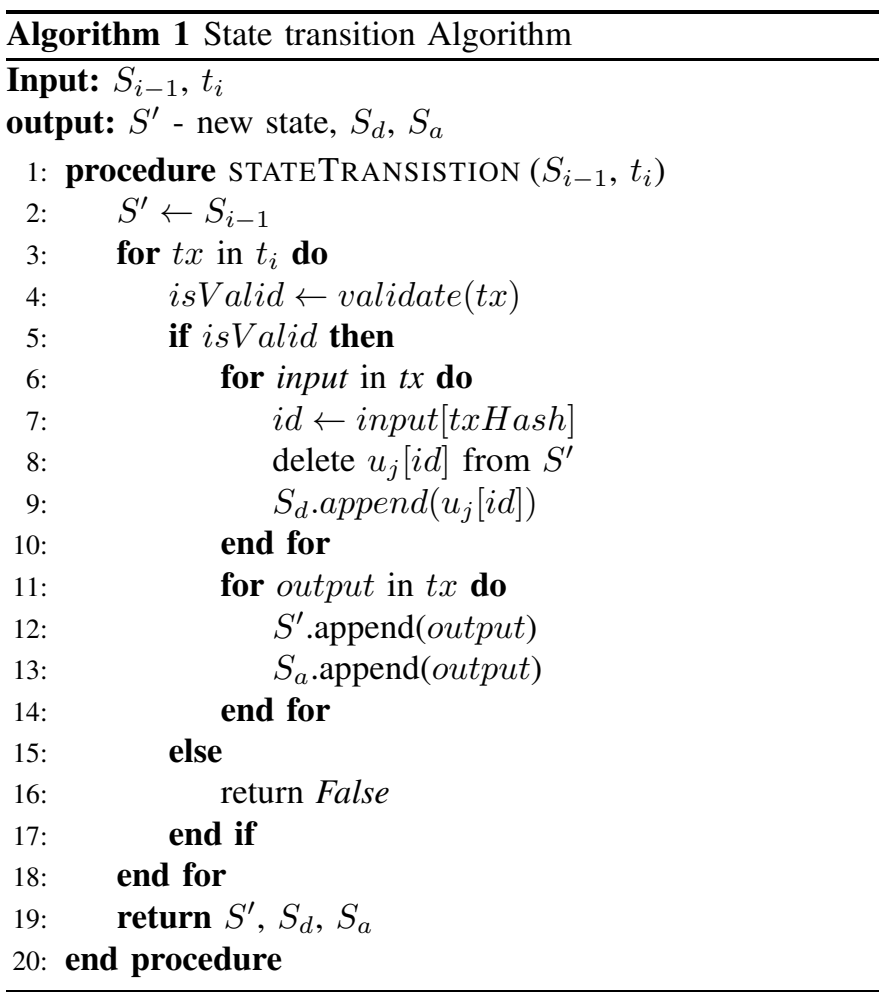

2) Modified PoW Algorithm: The modified Proof-of-Work function for mining a new block is described in Algorithm 2 . This PoW funtion includes Accumulator state $A_{i}$ along with other parameters into the block header for providng immutable blockchain state $S_{i}$. It also includes NI-PoE proofs $\left(\pi_{d}, \pi_{a}\right)$ for deletion and addition of the new set of elements $\left(S_{d}, S_{a}\right)$ to the state from the present transaction set $t_{i}$.

The NI-PoE proof $\pi_{d}$ is obtained from the BatchDel function [11] as a proof for deletion of the unspent transactions refered in the inputs of the set $t_{i}$. The BatchDel function deletes the sources of inputs $\left(S_{d}\right)$ from the accumulator state of the previous block $A_{i-1}$ and generates the NI-PoE proof $\pi_{d}$. The proof $\pi_{a}$ is also an NI-PoE proof generated from the BatchAdd function [11] for adding the outputs $\left(S_{a}\right)$ of set $t_{i}$

3) Block Validation Algorithm: We defined a validation function in Algorithm 3 to check the validity of $A_{i}, t_{i}, R_{i}, \pi_{d}$ and $\pi_{a}$ from the present state $S_{i-1}$, local chain $C_{i-1}$ and the received new block $\left(B_{i}\right)$. If $B_{i}$ is valid, the full node adds $B_{i}$ to $C_{i-1}$, otherwise discards the block.

\section{B. securePrune Protocol}

The protocol differs from the bitcoin protocol by issuing a snapshot of the UTXO set at regular intervals of every $\Delta_{s}$ blocks called snapshot interval. The miners while creating a new block as per the Algorithm 2 at a height $c \Delta_{s}$ $(c=1,2,3, \ldots)$ releases the snapshot along with the block $B_{p}$ created at that particular height. The snapshot conststs of an indetifier and a copy of the state $\left(S_{p}\right)$ consists of all the unspent tranasctions including the unspent transactions of the current block. The snapshot identifier is the accumulator state 


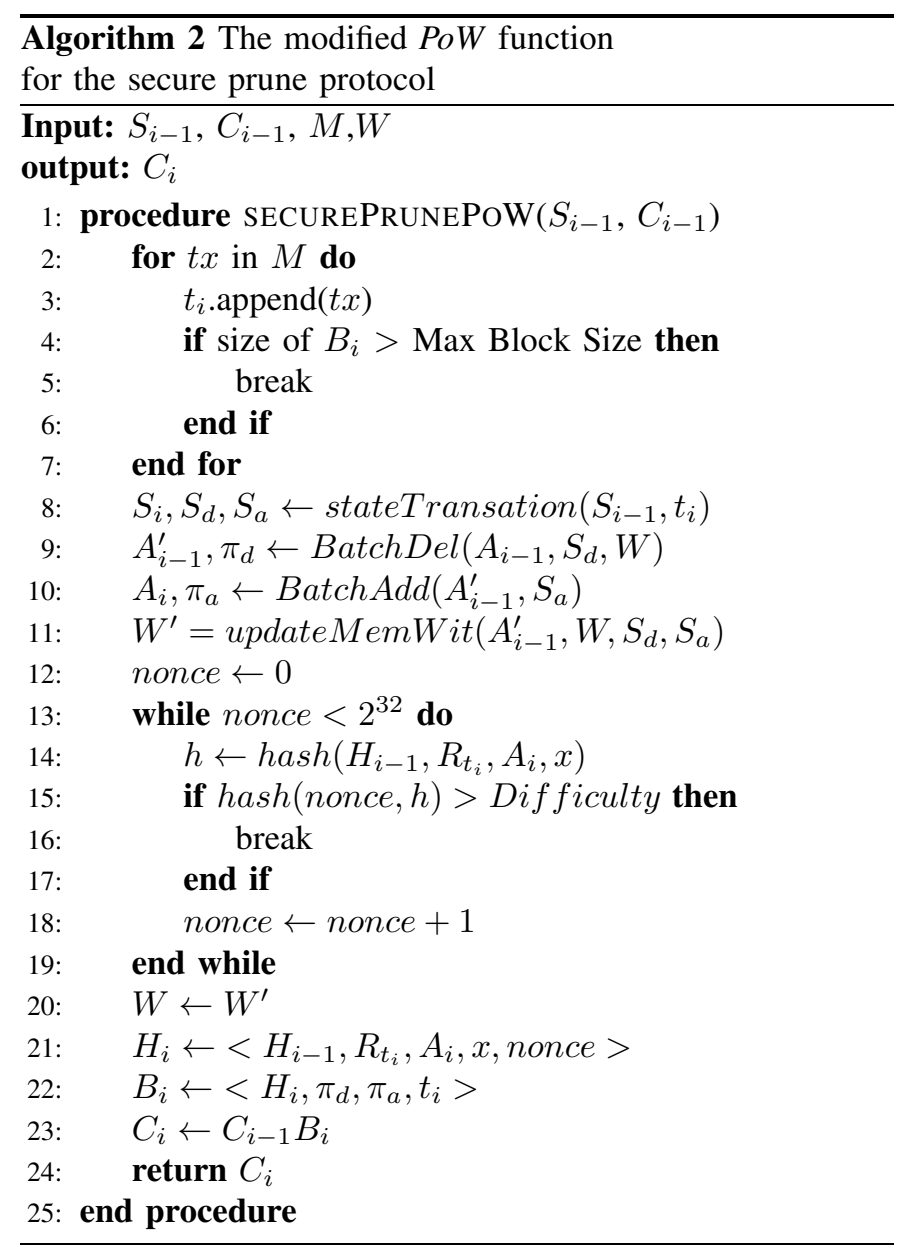

present in the block header of snapshot block $B_{p}$. The chain subsequent to the snapshot block $B_{p}$ is termed as the tailchain. The full node follows the Algorithm 3 for validation of a block created during $\Delta_{s}$ (present in the tailchain) by verifying the NI-PoE proofs $\pi_{d}$ and $\pi_{a}$, merkle root $R_{i}$ and transactions $t_{i}$.

The full nodes in the network prune all the historical blocks prior to the snapshot block $B_{p}$, provided that the block $B_{p}$ achieved $k$ number of confirmations from the tailchain blocks created in the network. The full nodes choose the tip of the longest chain similar to bitcoin [1] for deciding the number of confirmations on $B_{p}$. Suppose, more than one miner releases a snapshot at a height $p+c \Delta_{s}$, the snapshot with longest tailchain is a valid snapshot. Fig. 2 describes the overview of the secureprune protocol.

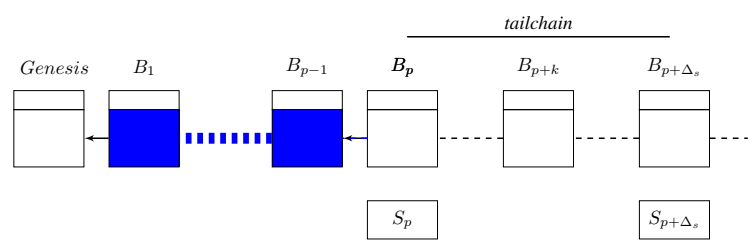

Fig. 2: Overview of securePrune protocol: The blue colour blocks are pruned after attaining a $k$ confirmations to block $B_{p}$.

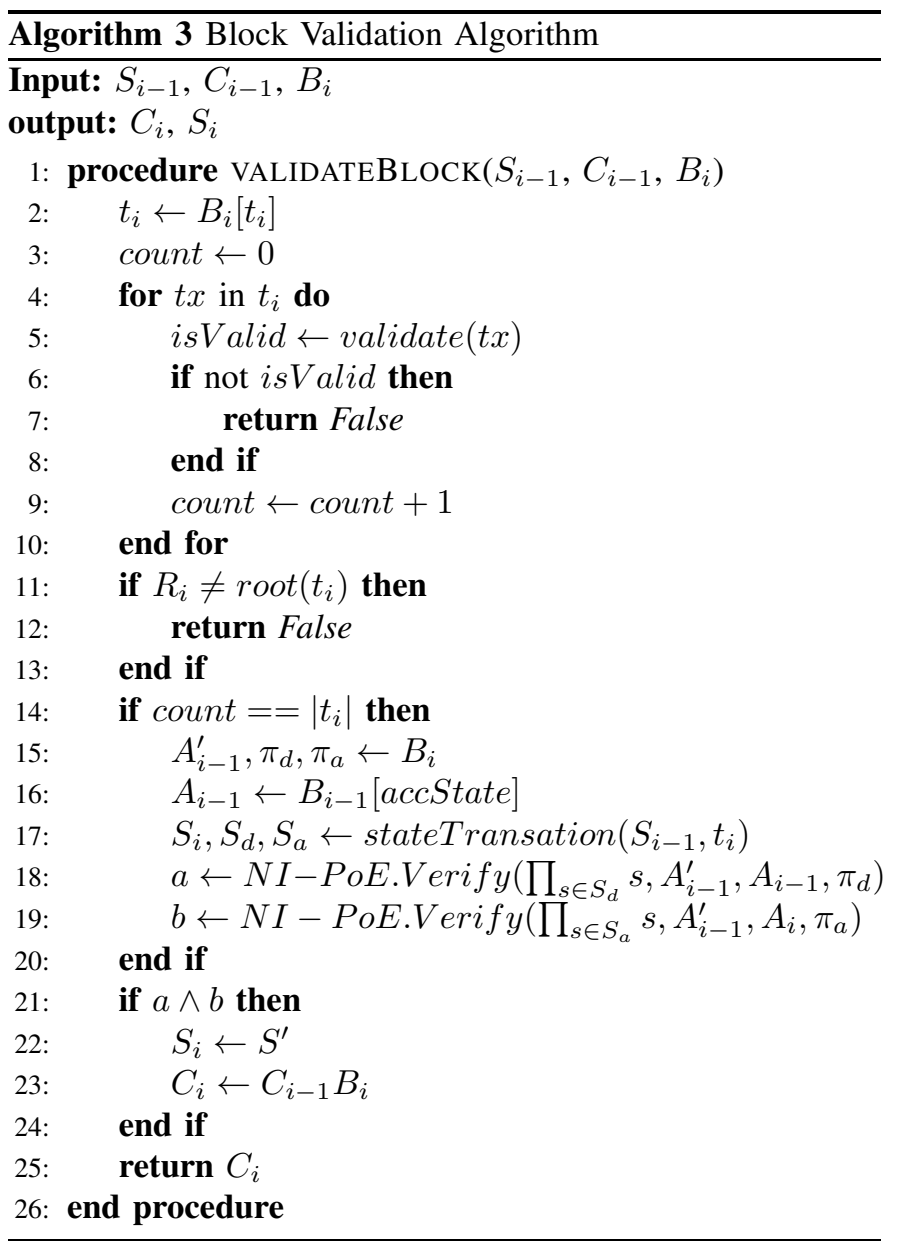

Lemma 5.1: Let $k$ be the number of confirmations required for a block with very low probability of double-spend to succeed by an attacker, then the number of confirmations required for a snapshot is also $k$.

Proof: If any attacker tries to modifies a transaction in a block, the hash of the block change as the merkle root is a function of all the transactions in a block [1]. The doublespend attack is the creation of a secret chain longer than the chain with the original transaction. So, an attacker needs to create a chain longer than the honest chain to modify any transaction of a particular block. The probability of doublespend to be succeed by an attacker with a fraction of hashrate $q$ and for a given number of confirmations is shown in [1] and [23].

Suppose, a miner in the network creates a block at height $p$ and has recieved $k$ number of confirmations. Let $B_{p+1}, B_{p+2}, \ldots, B_{p+k}$ are the blocks that confirms block $B_{p}$. A transaction in the block is said to be a part of the valid chain, if it has $k$ number of confirmations with very low probability of double-spend by an attacker.

Let - denotes the state transition function described in Alogorithm 11 the new state of a snapshot $S_{p}$ after $k$ number of blocks is appended to block $B_{p}$ is given by 


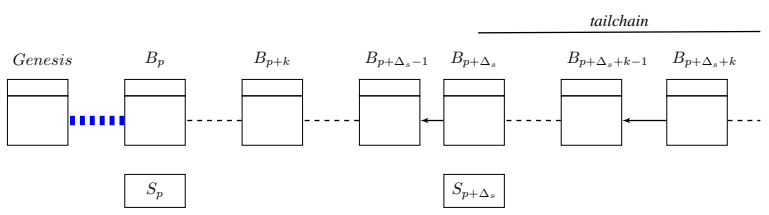

Fig. 3: The blue colured dashed line shows the pruned blocks in the chain

$$
\begin{aligned}
S_{p+k} & =S_{p+k-1} \bullet B_{p+k} \\
& =S_{p+k-2} \bullet B_{p+k-1} \bullet B_{p+k} \\
& =S_{p} \bullet B_{p+1} \bullet B_{p+2} \bullet \cdots \bullet B_{p+k}
\end{aligned}
$$

since the state is represented as a part of the PoW function in Algorithm 2 in terms of the accumulator state $A_{i}$ for a block $B_{i}$, the hash of the block changes with change in the state particularly snapshot.

Thus, the number of confirmations required for immutability of the snapshot is same as the number of confirmations required for a block against the double-spend attack.

\section{Size of the Blockchain in securePrune}

Fig. 3 shows the total blocks from block $B_{p}$ to the current height including $B_{p+\Delta_{s}}$, block at snap shot $S_{p+\Delta_{s}}$. Suppose, the nodes pruned the blocks till height $B_{p-1}$ after acheiving the required number of confirmations to snapshot $S_{p}$, then every node in the network stores the blocks from $B_{p}$ onwards till the current height.

Suppose, a miner broadcast a block into the network at $p+\Delta_{s}$ along with snapshot $S_{p+\Delta_{s}}$, then the total number of blocks till block height $p+\Delta_{s}+k-1$ are $\Delta_{s}+k$. At height $p+\Delta_{s}+k$, the nodes prune the blocks $B_{p}$ to $B_{p+\Delta_{s}-1}$. So, the total number of blocks stored with a node is upper bounded by $\Delta_{s}+k$.

\section{Synchronization of the Bootstrapping nodes}

The new node joining the network bootstrap in three steps - First, it obtains the most recent snapshot with the longest tailchain. Second, the new node downloads the entire headerchain since the genesis block and verifies the validity of the headerchain. Third, the node downloads the tailchain from its peers and validate all the blocks since the snapshot block to obtain its state.

Let $S_{p}$ is the most recent snapshot and a node joins the network at height $h$, then the state of the new node at height $h$ is obtained as

$$
S_{h}=S_{p} \bullet B_{p+1} \bullet \cdots \bullet B_{h}
$$

Let $b, c, s$ are the new nodes joining in bitcoin, coinPrune and securePrune respectively, then, $n_{b}, n_{c}, n_{s}$ are the number of blocks to be downloaded by nodes $b, c$ and $s$. The syncronization process depends on average download rate $(R)$ and average block validation Rate $\left(R_{v}\right)$ and block size $b$ (assuming
TABLE II:

Parameter values used for simulations

\begin{tabular}{|c|l|}
\hline Parameter & value \\
\hline$n$ & 1000 \\
\hline$n_{p}$ & 8 \\
\hline$\lambda$ & $1 / 600 \mathrm{blocks} / \mathrm{sec}$ \\
\hline$T_{p}$ & $30 \mathrm{msec}$ \\
\hline$b$ & $0.25 \mathrm{MB}$ \\
\hline$R$ & $10 \mathrm{Mbps}$ \\
\hline$k$ & 500 \\
\hline$\Delta_{s}$ & 1000 \\
\hline$R_{v}$ & $0.25 \mathrm{Mbps}$ \\
\hline$T_{\text {proofs }}$ & $0.35 \mathrm{sec}$ \\
\hline
\end{tabular}

a constant block size). The synchronization time required for these new nodes joining these networks are defined as follows

$$
\begin{aligned}
T_{b} & =n_{b} \times\left(\frac{b}{R}+T_{p}\right)+\frac{n_{b} \times b}{R_{v}} \\
T_{c} & =n_{c} \times\left(\frac{b}{R}+T_{p}\right)+\frac{n_{c} \times b}{R_{v}} \\
T_{s} & =n_{s} \times\left(\frac{b}{R}+T_{p}\right)+\frac{n_{s} \times b}{R_{v}}+n_{s} \times T_{\text {proofs }}
\end{aligned}
$$

The first term represents the downloading of the blocks from the existing node where as the second term denotes the validation of the blocks. In a securePrune network, the nodes need to validate the NI-PoE proofs in time $T_{\text {proofs }}$ for each block verification, whereas the number of blocks is less compared to the other two networks.

The bootstrap node, after obtaining its final state from the most recent snapshot and tailchain could acts as a full node to bootstrap the new joining nodes.

\section{RESULTS AND DISCUSSION}

Table $\Pi$ lists the values of the parameters used for generating the results in this section. See Table \ for a description.

We have conducted an event-driven simulation using python by generating events as per information propagation protocol [24] of bitcoin for propagating a block from miner to reach the entire network. The events are classified as inv - sending a new block hash invitation, getblock - requesting a new block, block - sending a block to its peers and addblock - adding a received block to its local copy of blockchain.

We have simulated for a duration of 70 days (equivalent to 10000) blocks with a block creation rate of $\lambda=\frac{1}{600}$ ( 1 block per every 10 minutes) similar to bitcoin block generation rate. We have chosen 13 nodes as miners with hash rates as per hash distribution shown in [25].

Fig. 4 show the time required for a full node to verify NIPoE proofs $\left(\pi_{d}\right.$ and $\left.\pi_{a}\right)$ with respect to the number of deleted sources of inputs $\left(\left|S_{d}\right|\right)$ and number of added outputs $\left(\left|S_{a}\right|\right)$. The verification time is very less $(\approx 0.35 \mathrm{sec}$ for 100 inputs and 100 outputs) compared to the block-creation time (600 $\mathrm{sec})$. 


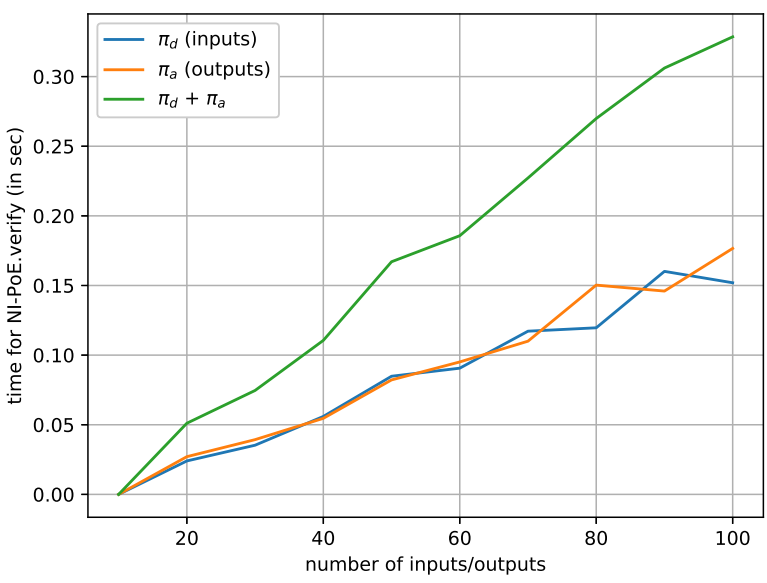

Fig. 4: Verification time of NI-PoE proofs Vs Number of inputs/outputs transactions per block

Fig. 5 show the total blockchain size of the nodes with respect to the block height. We have chosen the 1000 blocks between the two consecutive snapshots and 500 as number of confirmations (As the number of confirmations required for double-spend to succeed by an attacker (with fraction of hash rate $q=0.45$ ) with a probability $<10^{-4}$ is 462 as per the calculations given in [1]) for pruning the blocks prior to the snapshot. The nodes prune old blocks at heights $1000+500 c$ $(c=1,2,3, \ldots)$.

Denial-of-Service attack on coinPrune protocol: There is possible DOS attack on coinPrune [20] by the miners in the network. Since the coinprune requires a $k$ number confirmations out of the number of blocks in reaffirmation window to prune the blocks prior to the snapshot. If the minimum requirement of $k$ confirmations not attained for any snapshot, then the pruning could postponed to the next reaffirmation window. We chose miners $m_{D o S}=\{1,7,8,10,12,13\}$ arbitrarily as DoS attackers with collective hash rate of $0.377(\approx 38 \%)$ and the other miners participating in the reaffirmations are $m_{\text {reaffirm }}=\{2,3,4,5,6,9,11\}$ have collective hash rate of $0.623(\approx 62 \%)$. We chose 300 ( $60 \%$ of size of reaffirmation window) confirmations out of reaffirmation window of size 500 blocks. The coinPrune shows the pruning at block heights 3500 and 7500 . In this case, the pruning of the coinPrune node postponed a duration of 1000,2000 blocks due to DoS attack by the above mentioned miners even though the number of confirmations (300) chosen are less than the number of confirmations (500) chosen for securePrune block.

For values given in TABLE $\amalg$, the simulation results in Fig. 5 show the maximum storage of securePrune node is approaximately $400 \mathrm{MiB}\left(\left(\Delta_{s}+k\right) \times b\right)$ for a block size of $0.25 \mathrm{MiB}$, while the size of the bitcoin full node increases with block height. The reults show that $85 \%$ reduction in the the storage space of a securePrune node compared to bitcoin nodes.

Fig. 6 show the time required for a bootstrapping node to

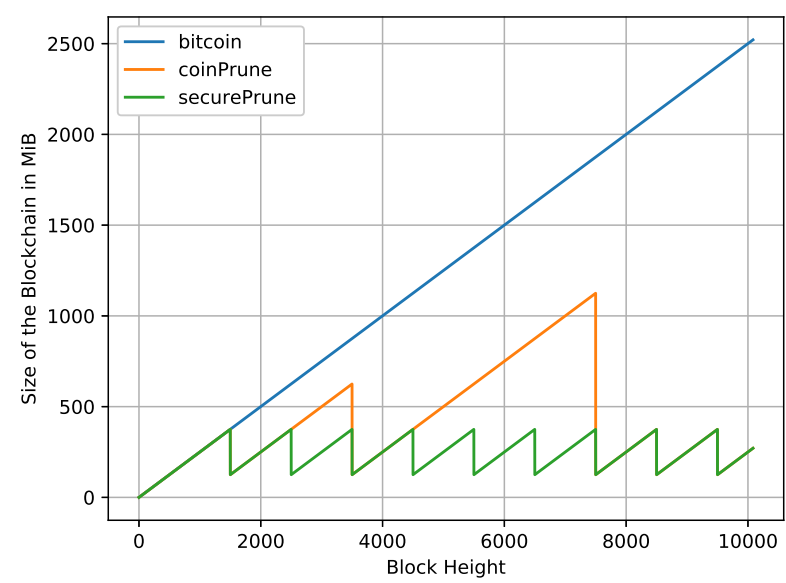

Fig. 5: Storage comparisons of a nodes in different protocols

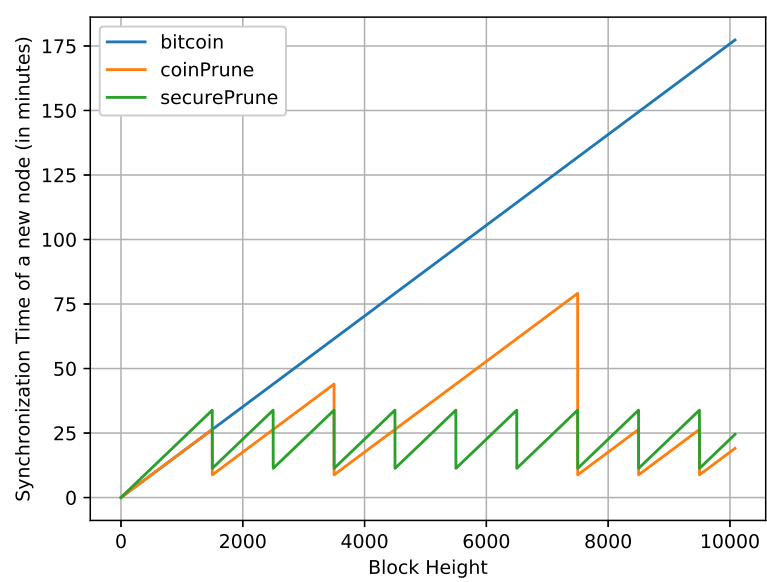

Fig. 6: The bootstrapping time of new nodes with respect to Block Height

synchronize with the existing nodes in the network. We hardcoded the block validation rate $R_{v}$ (depends on the processing speed of a node) and proofs verification time $T_{\text {proofs }}$ (from Fig. (4) in the simulation. The synchronization time linearly proportional to the number of blocks present in the chain at the time of joining a new node. The diiference in synchronization time of new nodes in coinPrune and securePrune after pruning is due to the extra time $\left(T_{\text {proofs }}\right)$ required for a new node in securePrune to verify the NI-PoE proofs. Fig. 6 show a significant reduction in synchronization time for a new node joining securePrune network compared to nodes joining the other two protocols.

Note: The results shown in Fig. 5 and Fig. 6 are obtained for different runs of simulations. 


\section{CONCLUSION AND FUtURE WORK}

In this paper, we show the periodic and secure pruning of the blocks prior to a certain block height based on the RSA accumulators. We proposed algorithms for generation of a block and validation of the block using NI-PoE proofs and accumulator state for securing the state of the blockchain along with transactions of the blocks. Through simulation results, we show the reduction in the storage space of a node in the proposed protocol which in turn reduce the synchronization time required to bootstrap a new node.

In future, we explore the exchanging of a snapshot from an existing node during the bootstrap of a new node while the state of the serving node changes with the creation of new blocks. We also consider the trade-off between the block generation, verification with efficient NI-PoE proofs and number of transactions in the block.

\section{REFERENCES}

[1] S. Nakamoto, "Bitcoin: A peer-to-peer electronic cash system," 2009. [Online]. Available: http://www.bitcoin.org/bitcoin.pdf

[2] ethereum/wiki, "A next-generation smart contract and decentralized application platform," 2015. [Online]. Available: https://github.com/ethereum/wiki/wiki/WhitePaper/

[3] E. Androulaki, A. Barger, V. Bortnikov, C. Cachin, K. Christidis, A. D. Caro, D. Enyeart, C. Ferris, G. Laventman, Y. Manevich, S. Muralidharan, C. Murthy, B. Nguyen, M. Sethi, G. Singh, K. Smith, A. Sorniotti, C. Stathakopoulou, M. Vukolic, S. W. Cocco, and J. Yellick, "Hyperledger fabric: A distributed operating system for permissioned blockchains," CoRR, vol. abs/1801.10228, pp. 1-15, 2018. [Online]. Available: http://arxiv.org/abs/1801.10228

[4] MedicalChain. (2018). [Online]. Available: https://medicalchain.com/en/

[5] R. J. Ali Dorri, Salil S. Kanhere and P. Gauravaram, "Blockchain for iot security and privacy: The case study of a smart home," in IEEE PERCOM Workshop On Security Privacy And Trust In The Internet of Things 2017. Kona, HI, USA: IEEE, March 2017, pp. 618-623.

[6] BitcoinCore, "Bitcoin Full Node," 2020. [Online]. Available: https://bitcoin.org/en/full-node

[7] S. Delgado-Segura, C. Pérez-Solà, G. Navarro-Arribas, and J. HerreraJoancomartí, "Analysis of the bitcoin utxo set," in Financial Cryptography and Data Security, A. Zohar, I. Eyal, V. Teague, J. Clark, A. Bracciali, F. Pintore, and M. Sala, Eds. Berlin, Heidelberg: Springer Berlin Heidelberg, 2019, pp. 78-91.

[8] BitcoinCore, "Bitcoin Source Code," 2020. [Online]. Available: https://github.com/bitcoin/bitcoin

[9] Blockchain, "Blockchain Luxembourg S.A." 2020. [Online]. Available: https://www.blockchain.com/charts/blocks-size

[10] N. Barić and B. Pfitzmann, "Collision-free accumulators and failstop signature schemes without trees," in Advances in Cryptology EUROCRYPT '97, W. Fumy, Ed. Berlin, Heidelberg: Springer Berlin Heidelberg, 1997, pp. 480-494.

[11] D. Boneh, B. Bünz, and B. Fisch, "Batching techniques for accumulators with applications to iops and stateless blockchains," in Advances in Cryptology - CRYPTO 2019, A. Boldyreva and D. Micciancio, Eds. Cham: Springer International Publishing, 2019, pp. 561-586.

[12] D. Perard, J. Lacan, Y. Bachy, and J. Detchart, "Erasure code-based low storage blockchain node," CoRR, vol. abs/1805.00860, 2018. [Online] Available: http://arxiv.org/abs/1805.00860

[13] S. Kadhe, J. Chung, and K. Ramchandran, "Sef: A secure fountain architecture for slashing storage costs in blockchains," CoRR, vol. abs/1906.12140, 2019. [Online]. Available: http://arxiv.org/abs/1906.12140

[14] M. Luby, "Lt codes," in The 43rd Annual IEEE Symposium on Foundations of Computer Science, 2002. Proceedings., 2002, pp. 271-280.

[15] R. K. Raman and L. R. Varshney, "Dynamic distributed storage for scaling blockchains," CoRR, vol. abs/1711.07617, 2017. [Online]. Available: http://arxiv.org/abs/1711.07617
[16] A. Shamir, "How to share a secret," Commun. ACM, vol. 22, no. 11, p. 612-613, Nov. 1979. [Online]. Available: https://doi.org/10.1145/359168.359176

[17] K. V. Rashmi, N. B. Shah, P. V. Kumar, and K. Ramchandran, "Explicit construction of optimal exact regenerating codes for distributed storage," in Proceedings of the 47th Annual Allerton Conference on Communication, Control, and Computing, ser. Allerton'09. IEEE Press, 2009, p. $1243-1249$

[18] A. S. Rawat, O. O. Koyluoglu, N. Silberstein, and S. Vishwanath, "Optimal locally repairable and secure codes for distributed storage systems," IEEE Transactions on Information Theory, vol. 60, no. 1, pp. 212-236, 2014.

[19] BitcoinCore, "Assumed-Valid vlocks," 2020. [Online]. Available: https://bitcoin.org/en/release/v0.14.0

[20] R. Matzutt, B. Kalde, J. Pennekamp, A. Drichel, M. Henze, and K. Wehrle, "How to securely prune bitcoin's blockchain," in 2020 IFIP Networking Conference (Networking), 2020, pp. 298-306.

[21] J. Camenisch and A. Lysyanskaya, "Dynamic accumulators and application to efficient revocation of anonymous credentials," in Advances in Cryptology - CRYPTO 2002, M. Yung, Ed. Berlin, Heidelberg: Springer Berlin Heidelberg, 2002, pp. 61-76.

[22] A. Shamir, "On the generation of cryptographically strong pseudorandom sequences," ACM Trans. Comput. Syst., vol. 1, no. 1, p. 38-44, Feb. 1983. [Online]. Available: https://doi.org/10.1145/357353.357357

[23] M. Rosenfeld, "Analysis of hashrate-based double spending," CoRR, vol. abs/1402.2009, pp. 1-13, February 2014. [Online]. Available: http://arxiv.org/abs/1402.2009

[24] C. Decker and R. Wattenhofer, "Information propagation in the bitcoin network," in IEEE P2P 2013 Proceedings. Trento, Italy: IEEE, Sep. 2013, pp. 1-10.

[25] Blockchain, "Hash Rate Distribution," 2020. [Online]. Available: https://www.blockchain.com/pools 